\title{
Effect on N Defect in Cu-doped III-nitride Semiconductors
}

\author{
Byung-Sub Kang*, Jae-Kwang Lee, Yong-Sik Lim, Kie-Moon Song, and Kwang-Pyo Chae \\ Department of Nano Science and Mechanical Engineering, Konkuk University, Chungju 380-701, Korea
}

(Received 16 June 2011, Received in final form 14 November 2011, Accepted 13 December 2011)

\begin{abstract}
We studied the effect on the electronic and magnetic properties of the $\mathbf{N}$ defect in clean and $\mathrm{Cu}$-doped wurtzite III-nitrides by using the first-principles calculations. When it is doped two $\mathrm{Cu}$ atoms in the nearest neighboring sites, the system of AIN, GaN, or InN with the $\mathrm{N}$ vacancy is energetically more favorable than that without the $\mathrm{N}$ vacancy site. When the $\mathrm{Cu}$ concentration increases, the total magnetic moment of a supercell becomes small. The ferromagnetism of $\mathrm{Cu}$ atom is very low due to the weak $3 d-3 d$ coupling. It is noticeable that the spinexchange interaction between the $\mathrm{Cu}-3 d$ and $\mathrm{N}$ defect states is important.
\end{abstract}

Keywords : Cu-doped III-nitrides, nitrogen defect, ferromagnetism

\section{Introduction}

The transition metal-doped III-nitrides diluted magnetic semiconductors (DMSs) have attracted intense attention because of their potential applications in spintronics devices which utilize both the charge and the spin of electrons to create new functional beyond conventional semiconductors. An ideal DMS should exhibit the ferromagnetism at room temperature for the practical applications and have a homogeneous distribution of the magnetic dopants. However, the formation of clusters or secondary phases between the magnetic dopants and host atoms will have a bad influence on the practical applications. The group III-nitrides AlN, GaN, InN and their alloys have been widely studied both theoretically and experimentally [1-10]. The Cr- and Mn-doped GaN semiconductors are now one of the most extensively studied classes of semiconductor materials. It has been reported that the Cr-doped AIN semiconductor films are ferromagnetic at temperature over $340 \mathrm{~K}$ [3]. Chen and coworkers have reported that the homogeneous $\operatorname{In}_{0.98} \mathrm{Cr}_{0.02} \mathrm{~N}$ film grown at $300^{\circ} \mathrm{C}$ shows the ferromagnetic properties with Curie temperature higher than $350 \mathrm{~K}$ [8]. Recently it has been reported that the nonmagnetic dopant $\mathrm{Cu}$ in $\mathrm{GaN}$ is a promising DMS free of magnetic precipitates [11]. They report that the $\mathrm{Cu}$-doped $\mathrm{GaN}$ favors ferromagnetic ground state which can be explained in terms of $p$ - $d$ hybridization

*Corresponding author: Tel: $+82-43-840-3627$

Fax: +82-43-851-4169, e-mail: kangbs@kku.ac.kr mechanism, and a Curie temperature around $350 \mathrm{~K}$ can be expected.

In the present paper, we studied systematically on the electronic and the magnetic properties for $\mathrm{Cu}$-doped group III-nitrides with a $\mathrm{Cu}$ concentration of $6.25-12.5 \%$, as well as the effects on the electronic properties of nitrogen vacancy were considered. The possibility of $\mathrm{Cu}$ as a nonmagnetic dopant to fabricate DMS based AIN was examined by first-principles calculations. As well known, the defects and the impurities are believed to play important roles in DMSs of wide semiconductor gaps. A recent theoretical study $[12,13]$ has been confirmed that the nitrogen vacancy should be the dominant defect in $\mathrm{GaN}$ in the whole range of Fermi-level positions in the band gap. We aimed to investigate the electronic structures and magnetism under different doping concentrations with (or without) the defect of nitrogen.

\section{Calculation Method}

The electronic and magnetic properties on the $\mathrm{Cu}-$ doped III-nitrides with the $\mathrm{Cu}$ concentration of $6.25 \%$ and $12.5 \%$ had been studied for a supercell of 32 atoms with one or two atoms substituted by $\mathrm{Cu}$ (the configuration of $\mathrm{Cu}$ site was specified in Fig. 4). The calculations had been performed by using a self-consistent full-potential linear muffin-tin orbital (FP-LMTO) method $[14,15]$ based on spin density functional theory with the local spin-density approximation (LSDA). The local part of the Wang and Perdew parameterization of the exchange-correlation 
energy was used [16]. The LMTO basis set and the charge density were expanded in terms of the spherical harmonics up to $l=6$ inside each muffin-tin sphere. The basis functions in the interstitial region were expanded by the smoothed Hankel functions [14]. The valence electrons were not assumed to have the spin-orbit coupling but had generated the self-consistent supercell potential by considering the scalar relativistic effects.

The atomic potentials were approximated by spherically symmetric potential, however the full charge density including all nonspherical terms was evaluated by Fourier transformation in the interstitial region. The charge density was determined self-consistently by using a gammacentered $4 \times 4 \times 4$ grid in the Brillouin zone. The improved tetrahedron method by Blöchl [17] had been chosen to improve the convergence of the electronic structure and total energy with respect to the number of $k$ points. Using $64 k$ points insured that the total energy and the magnetic moment converge with an accuracy of $10 \mathrm{meV} / \mathrm{cell}$ and $0.01 \mu_{\mathrm{B}} /$ atom, respectively. Broyden [18] and Pulay [19] strategies for charge-density mixing were used to accelerate convergence of charge and spin densities and potentials.

\section{Results and Discussion}

We found that the calculated equilibrium lattice parameters $a=3.108 \AA$ and $c / a=1.6009$ for the clean AlN. For GaN, they were $a=3.187 \AA$ and $c=5.183 \AA$. They were $a=3.480 \AA$ and $c=5.615 \AA$ for the clean InN. In our calculations, we performed a minimization of the total energy for the supercell volume when keeping the constant $c / a$ ratio. The calculated parameters are in agreement with the experimental and the calculated ones $[2,5$, 20-22].

Fig. 1 shows the densities of states (DOS) for the clean $\mathrm{AlN}, \mathrm{GaN}$, and $\mathrm{InN}$ with the vacancy at nitrogen site $\left(V_{N}\right)$. That is, it is a summation of DOS for $\mathrm{Al}, \mathrm{Ga}$, In, and $\mathrm{N}$ atoms. It shows the downward shift in energy by $4.2 \mathrm{eV}, 2.5 \mathrm{eV}$, and $1.4 \mathrm{eV}$ for $\mathrm{AlN}, \mathrm{GaN}$ and $\mathrm{InN}$, respectively, in comparison with complete structures. Filled DOS represents the states of the $V_{N}$ site. The defect states are not formed in mid bandgap. It is located at the top of valence band $\left(E_{v}\right)$ and the bottom of conduction band. In the case of AlN, it shows the p-type semiconductor character which introduces the acceptor defectstate. As can be seen in Fig. 1, the calculated band gaps of clean AlN, GaN, and $\mathrm{InN}$ are 3.7, 2.0, and $1.7 \mathrm{eV}$, respectively. These results of AIN and GaN are smaller than those of experimental values. On the other hand, for $\mathrm{InN}$, the band gap is larger than the experimental ones. In

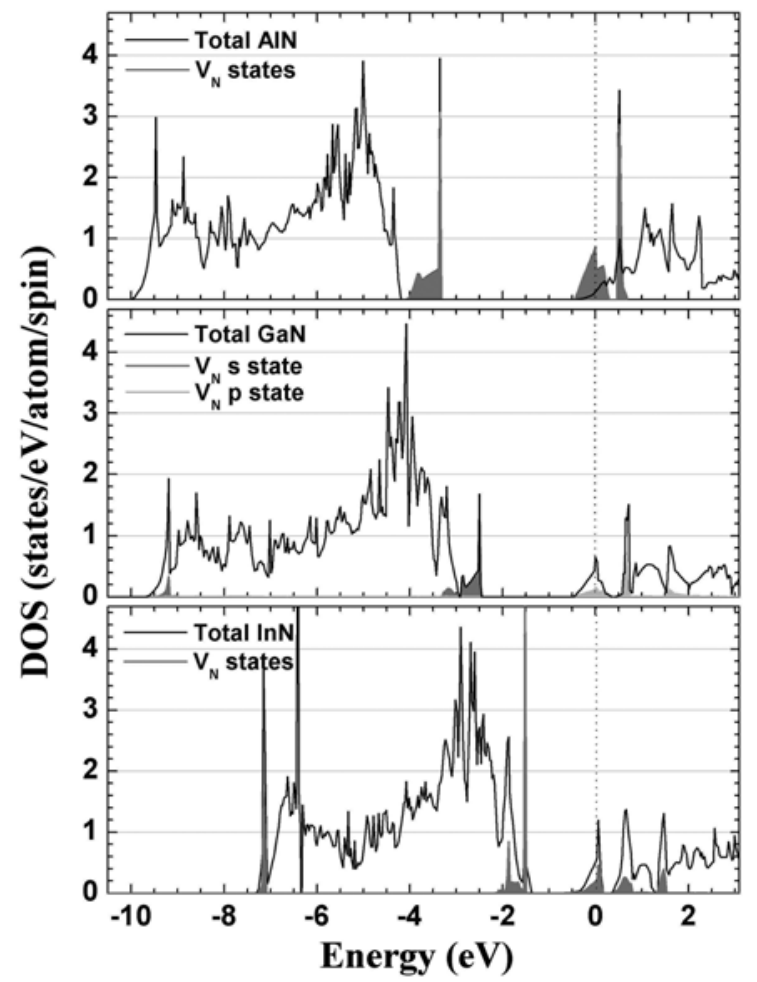

Fig. 1. Total DOS of AIN, GaN, and InN with $V_{N}$, Filled gray represents the $V_{N}$ states. The $E_{F}$ is set to zero.

Table 1. Formation energy of $\mathrm{N}$ vacancy $\left(\mathrm{E}_{\mathrm{f}}\right.$, in $\left.\mathrm{eV}\right)$, substitution energy $\left(\mathrm{E}_{\mathrm{s}}\right.$, in $\left.\mathrm{eV}\right)$, and total magnetic moments $(\mathrm{M} /$ cell, in $\left.\mu_{\mathrm{B}}\right)$ of supercell for the $\mathrm{X}_{0.9375} \mathrm{Cu}_{0.0625} \mathrm{~N}(\mathrm{X}=\mathrm{Al}, \mathrm{Ga}$, and $\mathrm{In})$ with the $\mathrm{N}$ vacancy. The values in the parenthesis represent the magnetic moment of $\mathrm{Cu}$ atom.

\begin{tabular}{cccc}
\hline \hline & AIN & GaN & InN \\
\hline $\mathrm{E}_{\mathrm{f}}$ & -11.413 & -8.899 & -6.581 \\
$\mathrm{E}_{\mathrm{s}}$ & -1.571 & -0.594 & -0.557 \\
$\mathrm{M} /$ cell & $0.75(0.43)$ & $0.50(0.25)$ & $0.38(0.19)$ \\
\hline
\end{tabular}

general, the value of band gap calculated by using the LSDA is less than half of the value obtained in the experiment.

Fig. 2(a) and 2(b) illustrate the differences in charge density, $\Delta \rho$, for the $\mathrm{Al}_{0.9375} \mathrm{Cu}_{0.0625} \mathrm{~N}$ and the $\mathrm{In}_{0.9375} \mathrm{Cu}_{0.0625} \mathrm{~N}$ with the $V_{N}$ site in the ground state. The $\Delta \rho$ is defined as $\rho\left(\mathrm{Cu}, \mathrm{XN},\left[V_{N}\right]\right)-\rho\left(\mathrm{XN},\left[V_{N}\right]\right)-\rho(1 \mathrm{Cu})$, where $\rho(1 \mathrm{Cu}$, $\left.\mathrm{XN},\left[V_{N}\right]\right)$ is the total charge density, and $\rho\left(\mathrm{XN},\left[V_{N}\right]\right)$ and $\rho(1 \mathrm{Cu})$ are the charge densities for $\mathrm{XN}(\mathrm{X}=\mathrm{Al}$ and $\mathrm{In})$ without [or with] the $V_{N}$ and an isolated $\mathrm{Cu}$ atom, respectively. The strong interaction between $\mathrm{Cu}$ and $\mathrm{N}$ atoms occurs due to the charge accumulation among neighboring $\mathrm{Cu}$ and $\mathrm{N}$ atoms. It shows the profile of moved charge in the site of a vacancy. In the case of AlN, the substitution energy between $\mathrm{Cu}$ and $\mathrm{Al}$ atoms is the 


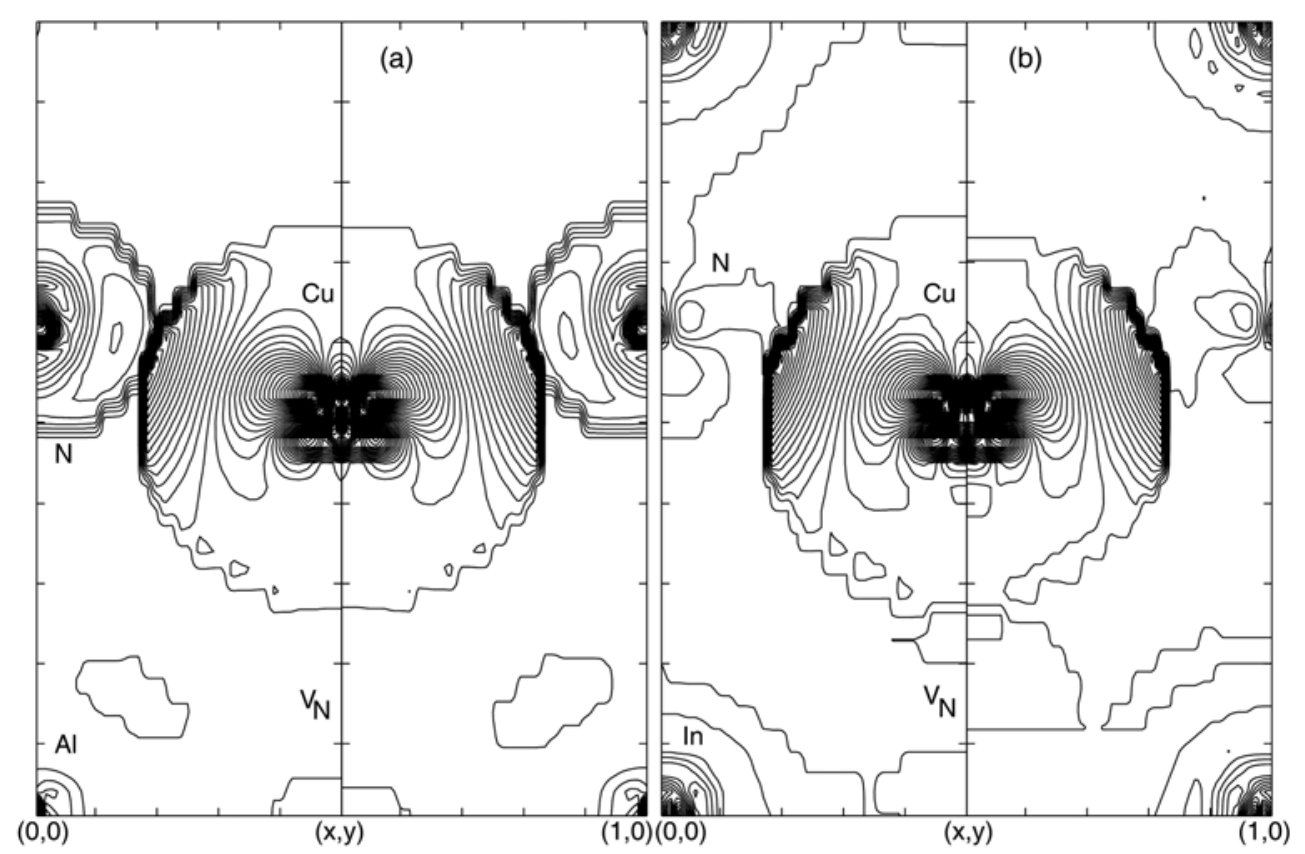

Fig. 2. Charge density difference $(\Delta \rho)$ for (a) the $\mathrm{Al}_{0.9375} \mathrm{Cu}_{0.0625} \mathrm{~N}$ and (b) the $\operatorname{In}_{0.9375} \mathrm{Cu}_{0.0625} \mathrm{~N}$ with the $V_{N}$. The contour is shown on the $(11 \underline{20})$ plane with intervals a $5.0 \times 10^{-4} \mathrm{e} /(\text { a.u. })^{3}$. The coordinate $(\mathrm{x}, \mathrm{y})$ corresponds to $(1 / 2,3 / 6)$. Here, the lattice parameter is set to 1 .

lowest, the vacancy formation energy also is the lowest. These results are listed in Table 1. The vacancy formation energy is defined as $\mathrm{E}_{\mathrm{tot}}(\mathrm{X}, \mathrm{N})-\mathrm{E}_{\mathrm{tot}}\left(\mathrm{X}, \mathrm{N}, V_{N}\right)-\mathrm{E}_{\text {tot }}(\mathrm{N})$. The substitution energies are defined as $\left(\mathrm{E}_{\text {tot }}\left(\mathrm{X}, \mathrm{N},\left[V_{N}\right]\right)-\right.$ $\left.\mathrm{E}_{\text {tot }}(\mathrm{X})\right)-\left(\mathrm{E}_{\text {tot }}\left(\mathrm{X}, \mathrm{Cu}, \mathrm{N},\left[V_{N}\right]\right)-\mathrm{E}_{\text {tot }}(\mathrm{Cu})\right)$, where $\mathrm{E}_{\text {tot }}(\mathrm{X}$, $\left.\mathrm{N},\left[V_{N}\right]\right)$ is the total energy of undoped-XN $(\mathrm{X}=\mathrm{Al}, \mathrm{Ga}$, and In) with (or without) the $\mathrm{V}_{\mathrm{N}}, \mathrm{E}_{\mathrm{tot}}\left(\mathrm{X}, \mathrm{Cu}, \mathrm{N},\left[V_{N}\right]\right)$ is the total energy of $\mathrm{Cu}$-doped system with (or without) the $V_{N}$. $E_{\text {tot }}(X, N)$ is the total energy of perfect $X N$ without the $\mathrm{V}_{\mathrm{N}}, \mathrm{E}_{\text {tot }}(\mathrm{X}), \mathrm{E}_{\text {tot }}(\mathrm{Cu})$, and $\mathrm{E}_{\text {tot }}(\mathrm{N})$ are that of an isolated $\mathrm{X}, \mathrm{Cu}$ and $\mathrm{N}$ atoms.

Fig. 3 shows the DOS for $\mathrm{X}_{0.9375} \mathrm{Cu}_{0.0625} \mathrm{~N}$ with the $V_{N}$ site. The $\mathrm{N}-2 s$ states in the group III-nitrides locate around $-14 \sim-12 \mathrm{eV}$. The lowest conduction band and the highest valence band originate mainly from $\mathrm{N}-2 p$ and $\mathrm{Cu}-3 \mathrm{~d}$ electrons. Fully occupied dopant states are generated just near the Fermi level $\left(E_{F}\right)$. The $E_{F}$ is located at the top of the valence band in the case of majority spinstates.

Fig. 4 shows the DOS for $\mathrm{X}_{0.875} \mathrm{Cu}_{0.125} \mathrm{~N}$ with the $V_{N}$ site. We can see that there is the difference in the crystal field splitting of $e$ and $t_{2}$ bands between the 'in-plane' and the 'out-of-plane' $\mathrm{Cu}$ sites. The structures of two $\mathrm{Cu}$ atoms in the $x y$-plane and of those positioned along the $c$ axis are referred to them as the 'in-plane' and the 'out-ofplane' sites, respectively. The atomic positions of 'inplane' and 'out-of-plane' sites are shown in the inset of Fig. 4. At high concentration of $\mathrm{Cu}$, the $t_{2}$-state is located

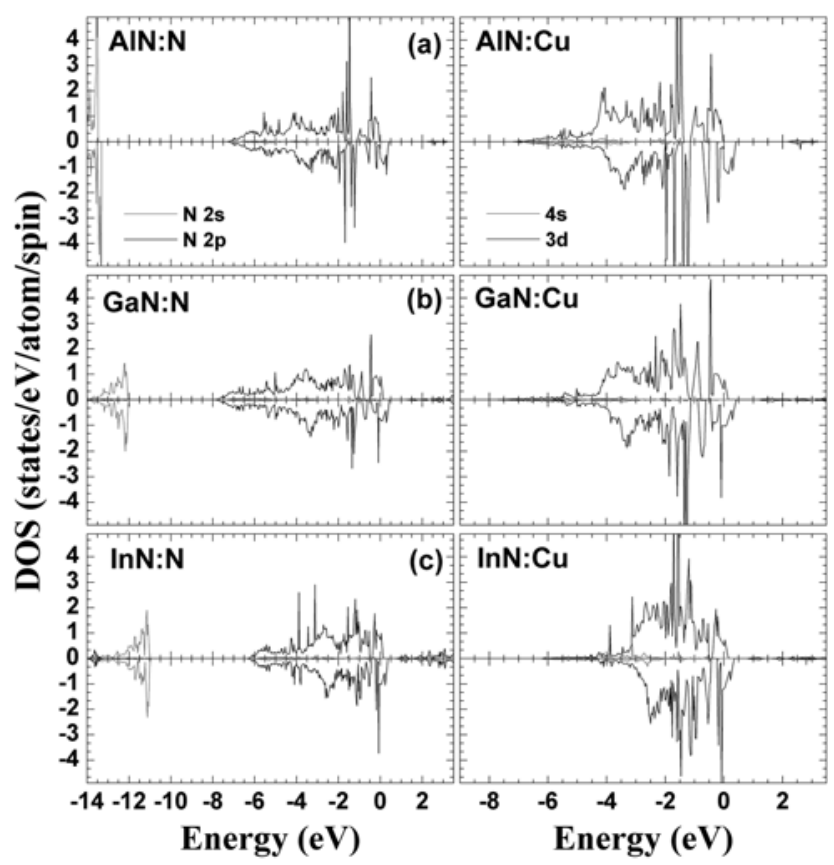

Fig. 3. Partial spin-polarized DOS of $\mathrm{N}$ and $\mathrm{Cu}$ in (a) $\mathrm{Cu}-$ doped AlN, (b) Cu-doped $\mathrm{GaN}$, and (c) Cu-doped $\mathrm{InN}$ with the $V_{N}$ and $\mathrm{Cu}$ concentration of $6.25 \%$. The $E_{F}$ is set to zero.

at the energy of $E_{F}-1.5 \mathrm{eV}$. The $e$-states are dominant and strongly localized just below the $E_{F}$. The crystal field splitting between $t_{2}$ and $e$ states in 'out-of-plane' $\mathrm{Cu}$ site of AlN is stronger than that of $\mathrm{InN}$. When the concen- 


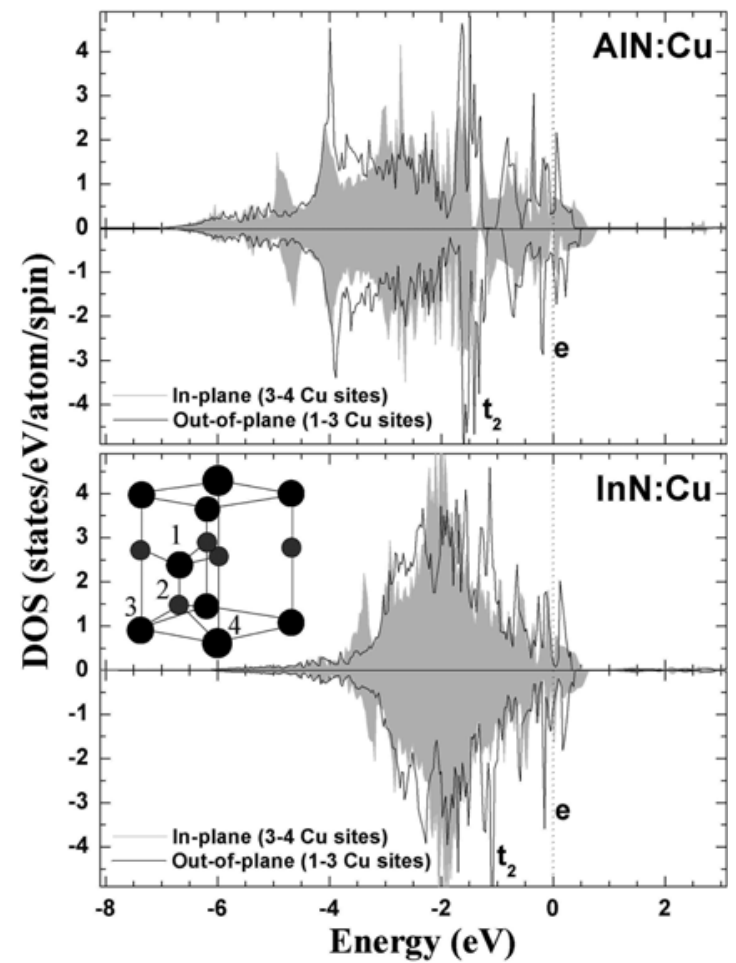

Fig. 4. Comparison between 'in-plane' (filled-gray) and 'outof-plane' (solid-line) sites for partial spin DOS of $\mathrm{Cu}-3 \mathrm{~d}$ in $\mathrm{Cu}$-doped AlN and $\mathrm{InN}$ with the $V_{N}$ and the $\mathrm{Cu}$ concentration of $12.5 \%$. The $E_{F}$ is set to zero. Wurtzite unitcell is shown in the inset. Number 1, 3, or 4 denotes the substituted $\mathrm{Cu}$ atom, number 2 denotes the $V_{N}$ site, the 3-4 site represents the 'inplane' $\mathrm{Cu}$ sites, and the 1-3 (or 1-4) site represents the 'outof-plane' $\mathrm{Cu}$ sites.

tration of $\mathrm{Cu}$ increases in $\mathrm{AlN}$ or $\mathrm{InN}$, the contribution in the ferromagnetism of $e$-levels is smaller than that of $t_{2}$ levels.

The $\mathrm{Cu}$ dopant orders ferromagnetically in the group III-nitrides. The ferromagnetic state is energetically more favorable than the nonmagnetic or antiferromagnetic state. As shown in Table 1, the $\mathrm{Cu}$ magnetic moments are 0.43, 0.25 , and $0.19 \mu_{\mathrm{B}}$, respectively, for $\mathrm{AlN}, \mathrm{GaN}$, and $\mathrm{InN}$ with the $\mathrm{Cu}$ concentration of $6.25 \%$. When the $\mathrm{Cu}$ concentration increases, it is introduced the strong hybridization between $\mathrm{N}-2 p$ and $\mathrm{Cu}-3 d$ electrons to reduce the magnetic moment of $\mathrm{Cu}$. For the 'in-plane' $\mathrm{Cu}$ site of $12.5 \%$, the $\mathrm{Cu}$ magnetic moments are 0.19 and $0.13 \mu_{\mathrm{B}}$ for $\mathrm{Cu}$-doped $\mathrm{AlN}$ and $\mathrm{InN}$, respectively. For both $\mathrm{Cu}-$ doped AIN and $\mathrm{InN}$, the substitution of $\mathrm{Cu}$ atom to the 'in-plane' site is energetically more favorable than that to the 'out-of-plane' site. These results are listed in Table 2.

The $V_{N}$ site in AlN, GaN, and $\mathrm{InN}$ produces a repulsive potential pushing the conduction band into the band gap, and it produces an attractive defect potential attracting
Table 2. Substitution energy $\left(\mathrm{E}_{\mathrm{s}}\right.$, in $\left.\mathrm{eV}\right)$ for the 'in-plane' and the 'out-of-plane' $\mathrm{Al}_{1-\mathrm{x}} \mathrm{Cu}_{\mathrm{x}} \mathrm{N}$ and $\mathrm{In}_{1-\mathrm{x}} \mathrm{Cu}_{\mathrm{x}} \mathrm{N}(x=0.125)$ with the $V_{N}$, total magnetic moments of supercell $\left(\mathrm{M} /\right.$ cell, in $\left.\mu_{\mathrm{B}}\right)$. The values in the parenthesis represent the average magnetic moment of $\mathrm{Cu}$ atoms. The superscripts of (a) and (b) represent the 'in-plane' and the 'out-of-plane' sites, respectively.

\begin{tabular}{ccccc}
\hline \hline & \multicolumn{2}{c}{ AIN } & \multicolumn{2}{c}{ InN } \\
\cline { 2 - 5 } & $\mathrm{E}_{\mathrm{s}}$ & $\mathrm{M} /$ cell & $\mathrm{E}_{\mathrm{s}}$ & $\mathrm{M} /$ cell \\
\hline $0.125^{(\mathrm{a})}$ & -3.127 & $0.67(0.19)$ & -1.064 & $0.48(0.13)$ \\
$0.125^{(\mathrm{b})}$ & -2.508 & $0.53(0.15)$ & -0.838 & $0.32(0.08)$ \\
\hline
\end{tabular}

levels of the valence state into the band gap. The defect site in AIN produces shallow acceptor states $\left(E_{v}+0.2 \mathrm{eV}\right)$. It induces the hybridization between the $V_{N}$ state and $\mathrm{Cu}$ $3 d$ states. The $\mathrm{Cu}$ magnetic moment is strongly reduced. The $\mathrm{Cu} 3 d-\mathrm{Cu} 3 d$ coupling becomes weak. Therefore, the $\mathrm{Cu}$ magnetic moment is very low. In addition, the $V_{N}$ site destroys the ferromagnetic coupling between the nearest neighboring two $\mathrm{Cu}$ atoms. The $\mathrm{Cu}$ dopant will combine easily with the site of $V_{N}$. Thus the control of $\mathrm{N}$ pressure plays important role in the design of high-performance devices.

\section{Conclusion}

We have systematically investigated the effects on the electronic and magnetic properties of $\mathrm{N}$ defect in clean or $\mathrm{Cu}$-doped wurtzite III-nitrides by using the full-potential linear muffin-tin orbital method. For the $\mathrm{Cu}$ concentration of $6.25 \%$ and $12.5 \%$, the ferromagnetic state is energetically more stable than the nonmagnetic and antiferromagnetic state. The site of $\mathrm{N}$ vacancy and the $\mathrm{Cu}$ dopant in $\mathrm{AlN}, \mathrm{GaN}$, and $\mathrm{InN}$ are strongly correlated. When there is a $V_{N}$ site, the system doped two $\mathrm{Cu}$ atoms in the nearest neighboring sites is energetically more favorable than that without the $V_{N}$ site. The $\mathrm{Cu}$ magnetic moment is strongly reduced due to the spin-exchange interaction between the $\mathrm{Cu}-3 d$ and $V_{N}$ states. When the $\mathrm{Cu}$ concentration increases in $\mathrm{AlN}, \mathrm{GaN}$, or $\mathrm{InN}$, the $\mathrm{Cu}$ site combines with the $V_{N}$ site easily. It may be formed a $\mathrm{Cu}+V_{N}$ state.

\section{Acknowledgment}

This work was supported by Konkuk University in 2011 (Dept. of Nano science and Mechanical engineering, Chungju).

\section{References}

[1] Q. Wang, A. K. Kandalam, Q. Sun, and P. Jena, Phys. 
Rev. B 73, 115411 (2006).

[2] X. Y. Cui, D. Fernandez-Hevia, B. Delley, A. J. Freeman, and C. Stampfl, J. Appl. Phys. 101, 103917 (2007).

[3] S. G. Yang, A. B. Pakhomov, S. T. Hung, and C. Y. Wong, Appl. Phys. Lett. 81, 2418 (2002).

[4] R. Frazier, G. Thaler, M. Overberg, B. Gila, C. R. Abernathy, and S. J. Peraton, Appl. Phys. Lett. 83, 1758 (2003).

[5] X. Y. Cui, J. E. Medvedeva, B. Delley, A. J. Freeman, and C. Stampfl, Phys. Rev. B 75, 155205 (2007).

[6] X. Y. Cui, J. E. Medvedeva, B. Delley, A. J. Freeman, N. Newman, and C. Stampfl, Phys. Rev. Lett. 95, 256404 (2005).

[7] R. F. C. Farrow and S. S. P. Parkin, Appl. Phys. Lett. 87, 172511 (2005).

[8] P. P. Chen, H. Makino, and T. Yao, J. Cryst. Growth 269, 66 (2004).

[9] A. Ney, R. Rajaram, S. S. P. Parkin, T. Kammermeier, and S. Dhar, Phys. Rev. B 76, 035205 (2007).

[10] M. Hashimoto, Y.-K. Zhou, M. Kanamura, and H. Asahi, Solid State Commun. 122, 37 (2002).

[11] R. Q. Wu, G. W. Peng, L. Liu, Y. P. Feng, Z. G. Huang, and Q. Y. Wu, Appl. Phys. Lett. 89, 062505 (2006).

[12] M. G. Ganchenkova and R. M. Nieminen, Phys. Rev. Lett. 96, 196402 (2006).

[13] Y. Li, W. Fan, H. Sun, X. Cheng, P. Li, X. Zhao, and M. Jiang, J. Solid State Chem. 183, 2662 (2010).

[14] S. Yu, Savrasov, Phys. Rev. B 54, 16470 (1996) and references therein.

[15] B. S. Kang, W. C. Kim, Y. Y. Shong, and H. J. Kang, J. Cryst. Growth 287, 74 (2006).

[16] Y. Wang and J. P. Perdew, Phys. Rev. B 43, 8911 (1991).

[17] P. E. Blöchl, O. Jepsen, and O. K. Andersen, Phys. Rev. B 49, 16233 (1994).

[18] C. G. Broyden, Math. Comput. 19, 577 (1965).

[19] P. Pulay, Chem. Phys. Lett. 73, 393 (1980).

[20] A. Zoroddu, F. Bernardini, and P. Ruggerone, Phys. Rev. B 64, 045208 (2001).

[21] S.-H. Wei and A. Zunger, Appl. Phys. Lett. 69, 2719 (1996).

[22] P. Rinke, M. Winkelnkemper, A. Qteish, D. Bimberg, J. Neugebauer, and M. Scheffler, Phys. Rev. B 77, 075202 (2008). 